\title{
Prediction of Location of Infarct-related Artery in acute Myocardial Infarction from Surface Electrocardiogram, its Clinical Importance and Therapeutic Strategy: A Review MOSTASHIRULHAQUE ${ }^{1}$, MD. SHAFIULALAM ${ }^{2}$, SABBIR JASHIMAHMED
, SUFIAKHATUN \\ ${ }^{1}$ Department of Cardiology, Anwer Khan Modern Medical College Hospital, ${ }^{2}$ Department of Medicine, BIRDEM General Hospital, ${ }^{3}$ Department of Cardiology, Bangabandhu Sheikh Mujib Medical University (BSMMU), Dhaka \\ Address of correspondence: Dr. Mostashirul Haque, Assistant Professor, Department of Cardiology, Anwer Khan Modern Medical College Hospital, Dhanmondi, Dhaka, Email: mustashir.haque@yahoo.com
}

\section{Introduction:}

Acute myocardial infarction is a common disease with serious consequences in mortality, morbidity, and cost to the society. ${ }^{1}$ Acute myocardial infarction (AMI) has always been a potential health problem due to the life-threatening complications. Coronary atherosclerosis plays a pivotal part as the underlying substrate in many patients. ${ }^{2} \mathrm{MI}$ results from prolonged myocardial ischemia precipitated in most cases by an occlusive coronary thrombus at the site of a pre-existing atherosclerotic plaque. ${ }^{1}$ Although coronary angiogram (CAG) is the gold standard to localize the site of coronary obstruction, ECG is an important tool in determining therapeutic strategy in acute coronary syndrome (ACS) in the setting of AMI. ${ }^{3}$

\section{Discussion:}

The diagnosis of infarct related artery (IRA) is very important with regard to the prediction of potential complications and subsequent therapeutic strategy in acute inferior myocardial infarction (AIMI). The IRA is right coronary artery (RCA) in $80 \%$ of cases, while it is left circumflex (LCX) in the rest. ${ }^{2}$ AIMI is sometimes complicated by hypotension or bradycardia, generally occurs in case of proximal RCA occlusion. So it is clinically important to determine proximal RCA occlusion to predict their potential complications and outline the therapeutic strategy in AIMI. ${ }^{3}$

Erdem Alim et al have studied sixty patients admitted to their center with diagnosis of AIMI.

Inclusion Criteria: acute inferior myocardial infarction is defined as presence of persistent ischemic chest pain (>30 $\mathrm{min})$, ST-segment elevation at least $1 \mathrm{~mm}$ in at least two of the inferior leads (L2, L3, aVF) and presence of at least two fold increase of cardiac biomarker (CK-MB) level compared to normal baseline value. Exclusion criteria: ST-segment elevation of $<1 \mathrm{~mm}$ in inferior leads,ST-segment elevation other than inferior leads, left ventricular hypertrophy(LVH), left bundle branch block(LBBB), presence of conditions causing ECG changes (electrolytes disturbances, myocarditis, pericarditis etc). ${ }^{3}$

Erdem Alim et al has shown the patients with the culprit lesion in the proximal portion were found to have a mean ST segment elevation $>12 \mathrm{~mm}$ but in the mid RCA and distal RCA portion were found $6 \mathrm{~mm}$ and $5 \mathrm{~mm}$, respectively. ${ }^{3}$

A ST segment elevation value of $>9 \mathrm{~mm}$ was found to have sensitivity and specificity of $91.3 \%$ and $94.6 \%$, respectively in the prediction of infarct related proximal RCA lesions. ${ }^{3}$

The presence of more severe ST segment elevation in the L3 compared to that in L2 was found to have a sensitivity of $99 \%$, specificity $100 \%$ and positive predictive value of $99 \%$ in the RCA occlusion in AIMI. ${ }^{4}$

Anterior MI carries worst prognosis of all infarct locations, mostly due to large infarct size. A study comparing outcomes from anterior and inferior infarctions (STEMI+NSTSEMI) founded that on average, patients with anterior MI had higher incidences of in-hospital mortality (11.9 vs $2.8 \%$ ), total mortality (27 vs $11 \%$ ), heart failure (41 vs 15\%) and significant ventricular ectopic activity (70 vs 59\%) and a lower ejection fraction on admission (38 vs 55\%) compared to patients with inferior MI. ${ }^{5,6}$

A number of studies have focused on the relationship between ECG changes and infarct related artery (IRA).

Birnbaum et al propounded ST segment depression in aVL lead- one of the sensitive and earliest parameter of AIMI. $^{7}$ 
Patients with an abnormal R wave in V1 (0.04 second in duration and $\mathrm{R} / \mathrm{S}$ ratio $>1$ in the absence of pre-excitation or right ventricular hypertrophy(RVH) with inferior or lateral $Q$ waves have an increased incidence of isolated occlusion of a dominant LCX without collateral circulation. ${ }^{8}$

Berry et al demonstrated that ST segment depression in both aVL and L1 leads was a marker of RCA occlusion in AIMI. ${ }^{9}$

Right-sided ST-segment elevation is indicative of acute right ventricular injury and usually indicates occlusion of the proximal RCA. Acute RVI projects an injury current in leads V1-V3, thereby simulating anterior infarction. ${ }^{10}$ Leads aVR may provide important clues to artery occlusion in MI. ${ }^{11}$

Zehender et al found an incidence of $27 \%$ of RVI associate with inferior MI based on electrographic criteria. ${ }^{22}$ In AIMI the more severe ST-segment elevation in L2 compared to that in L3 was found to have positive predictive value of $98 \%$ in the LCX occlusion. ${ }^{11}$ Inferior MI with ST depression in V1-V3 more associated with LCX occlusion (71\%) than RCA occlusion. ${ }^{12}$

Ratio of ST-segment depression in V3 to ST-segment elevation in L3 $<0.5$ predicts proximal RCA occlusion, 0.51.2 predicts distal RCA occlusion and $>1.2$ predicts LCX occlusion. $^{12}$

Inferior MI with ST-segment elevation in lateral leads (I, aVL, V5 \& V6) sensitive and specific marker for LCX occlusion. $^{8}$

Acute anteroseptal MI (with STE maximal in lead V1-V4), the following signs suggests proximal LAD occlusion: presence of ST-segment depression $>1 \mathrm{~mm}$ in inferior leads, ST-segment elevation in lateral leads (aVL), ST-segment elevation in lead aVR and right bundle branch block (RBBB). ${ }^{13}$ Left main or severe multi-vessel disease should be considered when leads aVR and V1 show ST-segment elevation with diffuse prominent ST depression in other leads. 11,14

STE in aVR of any magnitude is 43\% sensitive and 95\% specific for proximal LAD occlusion. ${ }^{13}$

$\mathrm{RBBB}$ in anterior $\mathrm{MI}$ is an independent marker of poor prognosis; this is due to the extensive myocardial damage involved rather the conduction disorder itself. ${ }^{13}$

Inferior lead ST-segment elevation accompany acute anterior MI suggesting either primary occlusion of a LAD that extends onto the inferior wall of LV (the type 111 or wrap-around $L A D$ ) or multi vessel disease (MVD) with jeopardized collaterals. ${ }^{15}$
Deep symmetrical precordial $\mathrm{T}$ wave inversions $>2 \mathrm{~mm}$ or biphasic $\mathrm{T}$ waves in V2-V3 indicating proximal LAD occlusion: a warning sign of imminent anterior infarction (Wellens syndrome). ${ }^{23}$ A new ECG sign of proximal LAD occlusions: Upsloping ST depression with symmetrically peaked $\mathrm{T}$ waves in the precordial leads; a "STEMI equivalent” indicating acute LAD occlusion (de Winter"s Twave). ${ }^{16}$

An isolated true posterior MI is unusual, less common (3$11 \%$ of infarcts). Posterior MI accompanies $15-20 \%$ of STEMIs usually occuring in the context of an inferior or lateral wall infarction. In such situation, the ECG changes of posterior wall infarction (Tall, broad R waves (>30ms) with ST-depression in V1-V2, upright T wave, dominant R wave( $\mathrm{R} / \mathrm{S}$ ratio $>1$ in $\mathrm{V} 2)$ are added. ${ }^{17}$

\section{Clinical Importance:}

Therefore, it is clinically important to determine the proximity of culprit lesion along RCA to predict the patients potentially at risk for AIMI related complications including brady-arrhythmia and severe hypotension. ${ }^{3}$ Right ventricular infarction is associated with increased risk of death, ventricular tachycardia or fibrillation and atrioventricular blocks. ${ }^{18}$ The frequency of VF and iatrogenic coronary dissection is higher when RCA is injected in the presence of a damped pressure tracing indicating ostial stenosis. Posterior extension of inferior or lateral infarct implies a much larger area of myocardial damage, with increased risk of LV dysfunction and death. ${ }^{17}$

It is pertinent to define the site of occlusion of LAD in the setting of AMI because proximal LAD occlusion needs more aggressive approach to revascularization to prevent extensive myocardial damage, development of sub-AV nodal conduction disturbances and occurrence of life threatening arrhythmias. ${ }^{10}$ Massive ST elevation with "tombstone" morphology is present throughout the precordial(V1-V6) and high lateral leads(1,aVL); this pattern is seen in proximal LAD occlusion and indicates a large territory infarction with poor LV ejection fraction and high likelihood of cardiogenic shock and death. ${ }^{5,13}$ In LMCA disease, most patients are symptomatic and high risk of $\mathrm{CV}$ events, since occlusion of this vessel compromises flow to at least $75 \%$ of $\mathrm{LV}$, unless it is protected by collateral flow or a patent bypass graft to either the LAD or LCX. ${ }^{6,11}$

\section{Therapeutic strategy:}

In patients presenting with inferior STEMI, who received fibrinolytic therapy, a routine invasive strategy with early CAG and intended revascularization, achieve a clinical outcome similar to an ischemia-guided strategy in which 
catheterization was based on the presence of myocardial ischemia and viability as demonstrated by stressmyocardial perfusion imaging (MPI). ${ }^{19}$ Pre hospital fibrinolytics with timely (6-24h) CAG with a view to revascularization (Pharmacoinvasive strategy) results in effective reperfusion in patients with early STEMI who could not undergo primary PCI ( $p P C I$ ) within 1 hour after first medical contact. ${ }^{20}$ In the strategic reperfusion early after MI (STREAM) trail, a pharmacoinvasive (PI) strategy was compared to the $p$ PCI in STEMI patient presenting within 3 hours after symptoms onset but unable to undergo pPCI within 1 hour, at one year mortality rates in the PI and pPCI arms were similar.

Isolated posterior infarction is an indication for emergent coronary reperfusion. ${ }^{17}$

The appropriateness of coronary artery bypass (CABG) surgery versus percutaneous coronary intervention (PCI) is predicted on clinical, anatomic and functional grounds. In the coronary artery surgery study (CASS), isolated left main coronary artery (LMCA) disease, left main equivalent (proximal LAD and LCX) CAD or three vessels CAD with LV dysfunction has demonstrated long term survival benefit from CABG surgery. Patients with an isolated high grade lesion of the proximal LAD have a significant better 4-year clinical outcome after off-pump CABG than after PCI. ${ }^{21}$ Major adverse cardiac events (MACES) occurred $27.5 \%$ after stenting and 9.8\% after surgery, freedom from angina was $67 \%$ after stenting and 85\% after surgery, need for anti-anginal medication was significantly lower after surgery compared to stenting. ${ }^{22}$ By contrast, in patient with relatively simple LMCA diseases, such as ostial / shaft LMCA disease, isolated LMCA disease with or without one or two vessel involvement and LMCA disease with low SYNTAX(Synergy between PCI with Taxus and Cardiac Surgery trail) score, PCI is an alternative and in some cases a preferred strategy to reduce surgical risks (e g. stroke). ${ }^{21}$ PCI is indicated in patients with suitable anatomy with refractory or lifestyle-limiting angina who have failed a course of optimum medical therapy (OMT).

\section{References:}

1. Naqvi MA, Ali M, Hakeem F et al. Correlation of severity of ST segment elevation in AIMI with the proximity of RCA disease. J Ayub Med Coll Abbottabad 2008; 20: 82-85.

2. Boersma E, Mercado N, Polderman D. Acute myocardial infarction. Lancet 2003;361 : 847-58.

3. Erdem A, Mehmet, Yilmaz B et al. The severity of ST-segment elevation in acute inferior MI: Does it predict the presence of a proximal culprit lesion along the RCA course ? Anadolu Kardiyol Derg 2007; 7:189-90.

4. Zimetbaum PJ, Krishnan S, Gold A et al. Usefulness of ST segment elevation in $\mathrm{L}_{\mathrm{III}}$ exceeding that of lead $\mathrm{L}_{\mathrm{II}}$ for indentifying the location of the occluded coronary artery in inferior MI. Am J Cardiol 1998;81:918-19.

5. Eskola MJ, NIkus KC, Homvang L et al.Value of 12 lead ECG to define the level of obstruction in acute anterior MI: correlation to CAG and clinical outcome in DANAMI-2 trail. Int J cardiol 2009;131:378-83.

6. Kosuge M, Kimura K, Ishikawa T et al. Predictors of LM or three vessels disease in patients who have ACS with NSTEMI. Am j Cardiol 2005; 95:1366-69.

7. Birnbaum Y, Sclarovsky S, Solodky A, et al. Prediction of the level of LAD obstruction during AMI (anterior wall) by admission electrogram. Am J Cardiol 1993 ; 72 : 823-26.

8. Herz I, Assali AR, Adier Y et al. New ECG criteria for predicting either RCA or LCX as the culprit artery in acute inferior MI. Am J cardiol 1997; 8:1343.

9. Berry C, Zalewski A, Kovach R et al. Surface electrogram in detecting of transmural MI during coronary artery occlusion. Am J cardiol 1989; 21-6.

10. Zimetbaum PJ, Josephson ME: Use of the electrocardiogram in acute myocardial infarction. N Eng J Med 2003; 348; 933.

11. Nikus KC, Sclarovsky S. ST-segment elevation in lead AVR of a sign of Left main disease - Perpetuating an error. Am J cardiol 2004; 94:542.

12. Kosuge M, Kimura K, Ishikawa $\mathrm{T}$ et al. New electrographic criteria for predicting the site of coronary artery occlusion in acute inferior MI. Am J cardiol 1998; 82:1318-322.

13. Engelen DJ, Gorgels AP, Cheriex EC, et al. Value of ECG in localizing the occlusion site in LAD artery in acute anterior MI. J Am Coll Cardiol 1999 ; 385-95.

14. Yamajii h, lwasaki K, Kusachi l et al. Prediction of acute LM artery occlusion by ST- segment elevation in lead $\mathrm{V}_{\mathrm{I}}$. J Am Coll Cardiol 2001; 38:1348.

15. Porter A, Sclarovsky S, Ben-gal $\mathrm{T}$ et al. Value of $\mathrm{T}$ wave direction with lead $\mathrm{L}_{\mathrm{III}}$ ST depression in acute anterior MI: ECG prediction of a wrapped LAD artery. Clinical Cardiol 1998; 21:562-66.

16. deWinter RJ, Verouden NJ.A new sign of proximal LAD occlusion. N Eng J Med 2008; 359:2071-73.

17. vanGorselen EO, Verheugt FW, Meursing BT et al. Posterior MI: the dark side of the moon. Neth Heart J 2007; 15.

18. Zehender MP, Kasper S, Kauder T. Right ventricular infarction as a independent predictor of prognosis after AMI. N Eng J Med 1993; 328:981-88.

19. Abdul Rahman S, Nammas W, Gamal A et al. Routine invasive versus ischemia -giuded strategy in patient with acute inferior STEMI who received fibrinolytic therapy: a prospective randomized controlled pilot trail. J invasive cardiol 2011; 23:316-21.

20. Armstrong PW, Gershlick AH, Goldstein P. Fibrinolyisis or Primary PCI in STEMI. N Eng J Med 2013; 368: 1379-87.

21. Park SJ, Park DW. Treatment of patients with left main CAD. Curr Treat Options Cardiovas Med 2011; 14:108-16.

22. Drenth DJ, Veeger VJ, Grandjean JG et al. Isolated high grade lesion of proximal LAD: a Stent or off-pump LIMA? Eurr J Cardiothoracic Surg 2004; 25:567-71.

23. de Zwaan C, Bar FW,Wellens HJJ. Characteristic ECG pattern indicating a critical stenosis high in LAD in patients admitted because of impending MI. American Heart Journal 1982; 103:730-36. 